\title{
Time to death, airway wall inflammation and remodelling in fatal asthma
}

\author{
A.L. James ${ }^{*} \#, \S$, J.G. Elliot ${ }^{*, \S}$, M.J. Abramson ${ }^{\star}$ and E.H. Walters ${ }^{+}$
}

\begin{abstract}
Fatal asthma is characterised pathologically by airway wall remodelling, eosinophil and neutrophil infiltration, accumulation of mucus in the airway lumen and smooth muscle shortening. The durations of fatal attacks of asthma show a clear bimodal distribution. Airway smooth muscle contraction and the accumulation of luminal mucus may contribute to death from asthma and relate to time to death.

The current authors have examined these two components in uninflated lung tissue in cases of fatal asthma from the second Victorian asthma mortality study. Based on time from onset of symptoms to death, cases fell into two distinct groups: short course $<3(1.5 \pm 0.6$ mean \pm SD) $h$; and long course $>8(12.3 \pm 5.9) \mathrm{h}$.

Short course cases had more muscle shortening, higher levels of salbutamol and higher ratios of neutrophils to eosinophils than long course cases, who tended to have more mucus in the lumen.

In conclusion, this study confirms the dichotomy of both time to death and the eosinophil/ neutrophil ratio in cases of fatal asthma. It suggests that in short course cases acute airway narrowing is due, predominantly, to bronchoconstriction despite higher blood levels of salbutamol. Mucus accumulation may be more important in long course cases.
\end{abstract}

KEYWORDS: Airway inflammation, fatal asthma, remodelling, salbutamol

A sthma is a common, chronic respiratory disease that causes substantial morbidity and mortality, much of which is potentially preventable. Patients with asthma have variable lung function and symptoms include breathlessness, chest tightness and wheeze. The airway pathology in asthma includes: 1) inflammation; 2) increased smooth muscle; 3) hyperplasia of submucosal mucous glands and goblet cells; 4) the laying down of extracellular matrix below the basement membrane; and 5) angiogenesis. All of which contribute to increased thickness of the airway wall [1-4].

Asthma symptoms are due to excessive airway narrowing, which may arise from increased (abnormal) smooth muscle shortening, exaggeration of the effects of muscle shortening due to encroachment of the thickened wall on the airway lumen, accumulation of mucus inside the airway lumen or a combination of all of these [5]. Fatal acute asthma is characterised pathologically by accumulation of mucus in the lumen, thickening of all compartments of the airway wall, eosinophilic and neutrophilic inflammatory infiltration of the airway wall and variable smooth muscle shortening (PMS) $[2,3,6,7]$.
A number of studies have demonstrated that there is a relationship between airway inflammation and the time course of a fatal attack of asthma [8-10]. It has been suggested that a short time course is immunologically distinct from a long time course of a fatal attack of asthma [9]. In all these studies, fatal attacks of short duration (short course) had a higher ratio of neutrophils to eosinophils in the airway wall than fatal attacks of long duration (long course). Intriguingly, none of these studies observed an overlap of the duration of attacks between the short course and long course cases, suggesting different mechanisms. An alternative explanation was offered by CARroll et al. [10] who showed that in the short course cases, not only was there an increase in the neutrophil/eosinophil ratio (as previously observed), but also an inverse relationship of time to death and the area of the submucosal mucous glands. They suggested that the short course cases died more quickly due to a sudden stimulus acting on more severe airway pathology, and that the relative neutrophilia reflected the early influx of neutrophils preceding the later influx of eosinophils that is seen following many inflammatory stimuli [11].
AFFILIATIONS

*West Australian Sleep Disorders Research Institute, Sir Charles Gairdner Hospital, Nedlands, \#School of Medicine and Pharmacology, University of West Australia, Perth,

"Dept of Epidemiology and Preventive Medicine, Central and Eastern Clinical School, Monash University, Melbourne, and ${ }^{+}$Cardio-Respiratory Research Group University of Tasmania, Hobart, Australia.

${ }^{\S}$ Authors contributed equally to this manuscript.

\section{CORRESPONDENCE}

A.L. James

West Australian Sleep Disorders

Research Institute

Sir Charles Gairdner Hospital Nedlands

Western Australia 6009

Australia

Fax: 0893462034

E-mail: ajames@it.net.au

Received

December 212004

Accepted after revision:

June 022005

\section{SUPPORT STATEMENT}

A.L. James is supported by a National Health and Medical Research Council of Australia Practitioner Fellowship (Canberra Australia). The Victoria Asthma Mortality Study (Victoria, Australia) was supported by the National Health and Medical Research Council of Australia. 
Epidemiological studies in the late 1980s and early 1990s from New Zealand and Canada suggested that excessive use of bronchodilator medications was associated with an increased risk of death from asthma $[12,13]$, although it was difficult to exclude the confounding effects of severity of the underlying asthma pathology [14]. In 1993, Monash University (Melbourne, Australia) and the Victorian Institute of Forensic Medicine (VIFM; Victoria, Australia) commenced an epidemiological study of asthma management and mortality in the state of Victoria, Australia. The study examined morbidity from asthma, compared drug levels in asthma deaths with those in severe asthma control cases and estimated the risk of death associated with particular patterns of asthma management and specific medications. Post mortem examination of the lungs was undertaken in all cases of asthma death reported to the VIFM. Substantial histories of asthma morbidity were observed in both cases and controls [15]. Blood salbutamol levels were significantly higher in cases than in controls, even after adjusting for age, sex, mode of salbutamol administration, ambulance attendance and the underlying severity of asthma.

Death during an asthma attack might result from sustained, severe airway narrowing due to PMS in combination with increased thickness of the airway wall and/or to the accumulation of mucus inside the airway lumen. The artefact introduced by the inflation of the lung specimens during fixation has confounded detailed quantitative assessment of the contribution of these factors in studies of fatal asthma. This may reverse PMS and dislodge mucus from the airway lumen.

As part of this study, lung tissues were fixed without inflation. The current authors, therefore, were able to examine PMS and mucus accumulation in relation to time to death and post mortem blood levels of salbutamol from cases of fatal asthma. The authors hypothesised that increased muscle shortening would be present in fatal attacks of asthma of short duration, whereas increased mucus accumulation would be associated with a longer course of a fatal attack of asthma.

\section{METHODS}

Cases of fatal asthma were from the second Victorian Asthma Morbidity Study [15]. All cases from this study were $<60$ yrs of age, had asthma as the primary cause of death on the death certificate and were reviewed by two clinicians to confirm that asthma was indeed the underlying cause of death. Medications used in the month prior to death were ascertained by administering the South Australian asthma mortality questionnaire to the next of kin [16]. The duration of the fatal attack of asthma was obtained from death certificates, coronial records and police and post mortem reports. The $\beta_{2}$-agonist assays were undertaken on whole blood or plasma specimens analysed by gas chromatography-mass spectrometry. A solid phase extraction procedure was employed, giving a limit of quantitation of $1 \mathrm{ng} \cdot \mathrm{mL}^{-1}$ for each analyte, in $1 \mathrm{~mL}$ of blood and plasma [17].

Tissue blocks were taken at random from several sites in the lung at post mortem examination at the VIFM. The tissue was fixed by immersion in $10 \%$ buffered formalin solution for $>24 \mathrm{~h}$ and then passed through a series of graded alcohols and embedded in paraffin wax. Sections were cut at $5 \mu \mathrm{m}$, mounted on glass slides, stained with haematoxylin and eosin and covered with a glass coverslip.

On airways cut in transverse section, the basement membrane perimeter $(\mathrm{Pbm})$ was used to define airway size. The inner (WAi) and outer (WAo) airway wall area and the area of smooth muscle (ASM) were measured. The percentage of PMS was calculated by comparing the measured outer perimeter (fig. 1a) of the ASM with its calculated relaxed perimeter [18] The amount of luminal mucus (including cellular debris) in the airway (fig. 1b) was measured and expressed as the mucus occupying ratio [19]. The number of neutrophils and eosinophils were counted in the WAi and WAo and expressed as neutrophil/eosinophil ratio. Alveolar attachments to the airway wall were counted and divided by the adventitial perimeter of the airway wall, minus the length of any section that was contiguous with an adjacent pulmonary vessel.

Case groups were categorised using time to death as short or long course. Statistical analysis was undertaken by running normality and equal variance tests. Differences between
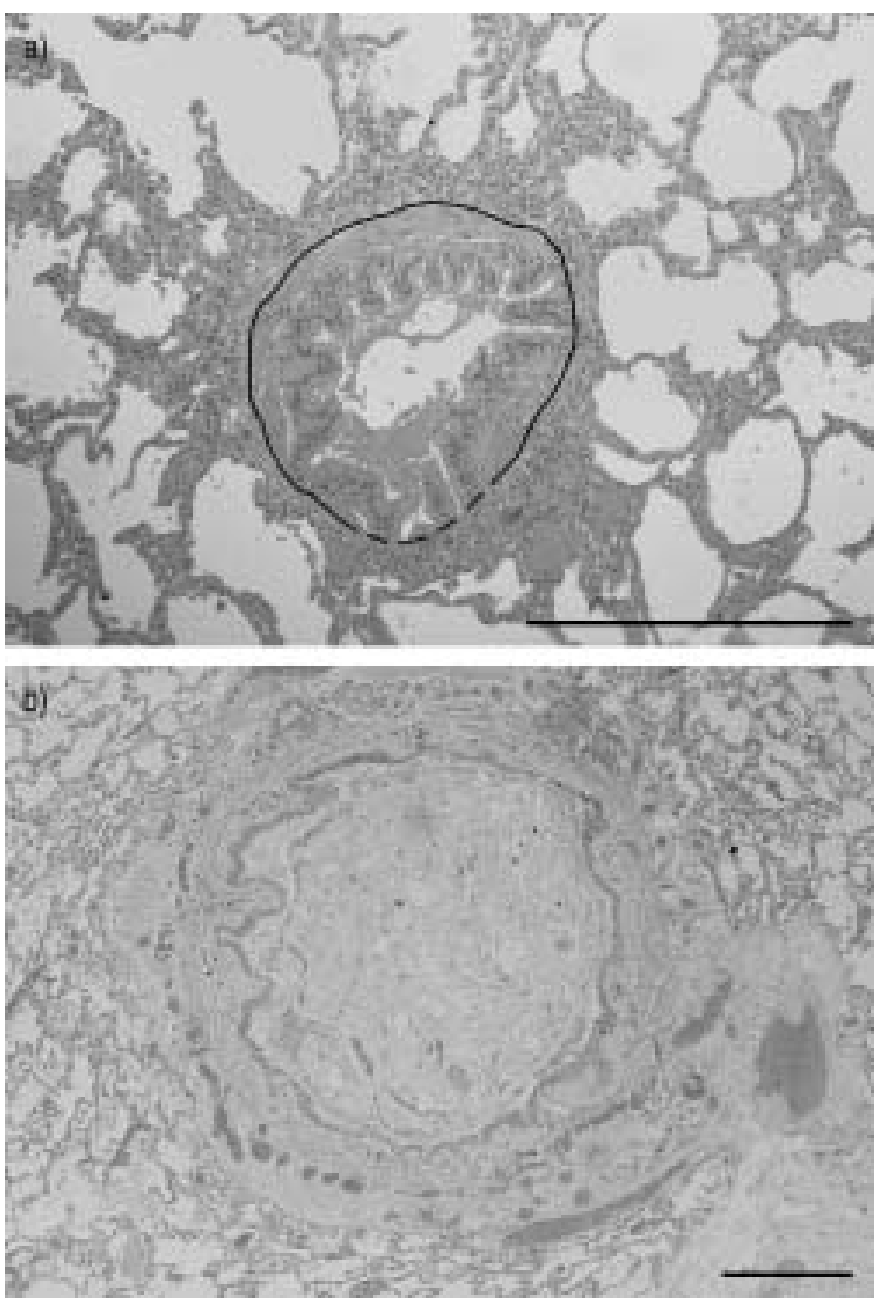

FIGURE 1. Transverse sections of membranous airways showing a) a highly contracted airway from a short course case with the perimeter of the smooth muscle highlighted (Scale bar $=0.5 \mathrm{~mm}$ ), and b) mucus accumulation occluding the airway in a long course case (Scale bar $=0.5 \mathrm{~mm}$ ). 
groups were compared using Mann-Whitney U-tests for variables that were not normally distributed and unpaired $t$ tests for normally distributed variables. Results are expressed as mean $\pm \mathrm{SD}$. Blood salbutamol levels were log transformed prior to analysis. All measurements were made by the one observer who was blinded to the case classification.

\section{RESULTS}

A total of 24 cases were identified by the Victorian Asthma Mortality Study panel as having asthma as the primary cause of death and had both questionnaire data and post mortem tissue available. Of these, 19 cases had airways that were cut in transverse sections and were suitable for morphometric analysis. The mean $\mathrm{Pbm}$ (airway size) was $4.49 \pm 1.9 \mathrm{~mm}$ for the entire group. One case did not have data regarding salbutamol levels, and in three cases the time to death was not available. Measurements of airway dimensions in the authors' laboratory had a coefficient of variation of $<5 \%$ [20].

The 16 cases where time to death was available fell into two distinct categories, based on time to death from the onset of the fatal attack, short course $(<3 \mathrm{~h}) 1.5 \pm 0.6 \mathrm{~h}$ and long course $(>8 \mathrm{~h}) 12.3 \pm 5.9 \mathrm{~h}$ (table 1$)$. There were no cases that died between 3-8 h. Groups were similar for age, weight and height, although there was a trend to more males in the short course cases. Data regarding severity of asthma was available for 12 cases. Markers of severity were not significantly different between the long course and short course cases with regard to hospitalisation in the last 12 months $(25 \%$ and $25 \%$, respectively), use of prednisolone in the last 12 months (33\% and $37 \%$, respectively), general practitioner score for overall severity ( $2.3 \pm 1.3$ and $2.4 \pm 1.1$, respectively) and symptom

\begin{tabular}{|c|c|c|}
\hline & $\mathbf{S C}$ cases $<\mathbf{3} \mathbf{h}$ & LC cases $>8 \mathrm{~h}$ \\
\hline Time to death hrs & $1.5 \pm 0.6$ & $12.3 \pm 5.8$ \\
\hline Case $\mathrm{n}$ & 10 & 6 \\
\hline Age yrs & $19.5 \pm 9.9$ & $23.3 \pm 12.8$ \\
\hline Male/female & $8 / 2$ & $3 / 3$ \\
\hline Weight kg & $53 \pm 18$ & $60 \pm 26$ \\
\hline Height $\mathrm{cm}$ & $163 \pm 22$ & $157 \pm 22$ \\
\hline Salbutamol $\mathrm{ng} \cdot \mathrm{mL}^{-1}$ & $153 \pm 161$ & $17 \pm 14^{*}$ \\
\hline Airway n & 71 & 31 \\
\hline Airway $n$ per case & $7.1 \pm 5.6$ & $5.2 \pm 4.1$ \\
\hline $\mathrm{Pbm} \mathbf{m m}$ & $3.9 \pm 1.3$ & $4.4 \pm 2.1$ \\
\hline Wall area inner $\mathrm{mm}^{2}$ & $0.07 \pm 0.03$ & $0.11 \pm 0.07$ \\
\hline Wall area outer $\mathbf{m m}^{2}$ & $0.20 \pm 0.14$ & $0.44 \pm 0.45$ \\
\hline Area smooth muscle $\mathrm{mm}^{2}$ & $0.03 \pm 0.01$ & $0.05 \pm 0.03$ \\
\hline NER in the inner wall & $0.47 \pm 0.7$ & $0.31 \pm 0.1 * *$ \\
\hline NER in the outer wall & $0.41 \pm 0.1$ & $0.26 \pm 0.1^{*}$ \\
\hline Muscle shortening \% & $23 \pm 7$ & $14 \pm 5^{\star}$ \\
\hline Mucus occupying ratio & $0.14 \pm 0.1$ & $0.23 \pm 0.8$ \\
\hline Alveolar attachments $\mathrm{n}$ per $\mathrm{mm}$ & $0.16 \pm 0.03$ & $0.18 \pm 0.07$ \\
\hline
\end{tabular}

Data are presented as mean \pm SD unless otherwise stated. SC: short course; LC: long course; Pbm: basement membrane perimeter; NER: neutrophil/ eosinophil ratio. ${ }^{*}: p<0.05 ;{ }^{* *}: p<0.01$ frequency score in the last 12 months $(4.3 \pm 2$ and $2.0 \pm 1.4$, respectively; $\mathrm{p}=0.072$ ).

Short course cases had a higher ratio of neutrophils to eosinophils in the WAi $(p=0.008)$ and WAo ( $p=0.05$; fig. 2$)$, more PMS $(p=0.01)$ and higher blood salbutamol levels at the time of autopsy $(p=0.05)$, compared with long course cases. In the short course cases, salbutamol levels ranged from 0 $511.6 \mathrm{ng} \cdot \mathrm{mL}^{-1}$, six of the 10 cases were $>80 \mathrm{ng} \cdot \mathrm{mL}^{-1}$ and four cases were $>200 \mathrm{ng} \cdot \mathrm{mL}^{-1}$. In the long course cases, salbutamol levels ranged from $0-37.9 \mathrm{ng} \cdot \mathrm{mL}^{-1}$ with only two cases $>20 \mathrm{ng} \cdot \mathrm{mL}^{-1}$. The mucus occupying ratio was $0.14 \pm 0.1$ in the short course cases and $0.23 \pm 0.8$ in the long course cases $(p=0.087)$. Of the cases with reliable information regarding the use of inhaled or oral corticosteroids in the last 12 months $(n=8), 66 \%$ of the long course and $80 \%$ of the short course cases were using inhaled corticosteroids. There was no significant difference between the groups with regard to the number of alveolar attachments around the airway perimeter, ASM and airway wall thickness (table 1). No correlation was apparent between salbutamol levels and muscle shortening percentage (fig. 3).

\section{DISCUSSION}

In the current study of fatal cases of asthma, it was found that the duration of the fatal attack distinguished two groups of cases with no overlap. Cases with a short course of the fatal attack had a higher neutrophil to eosinophil ratio than those
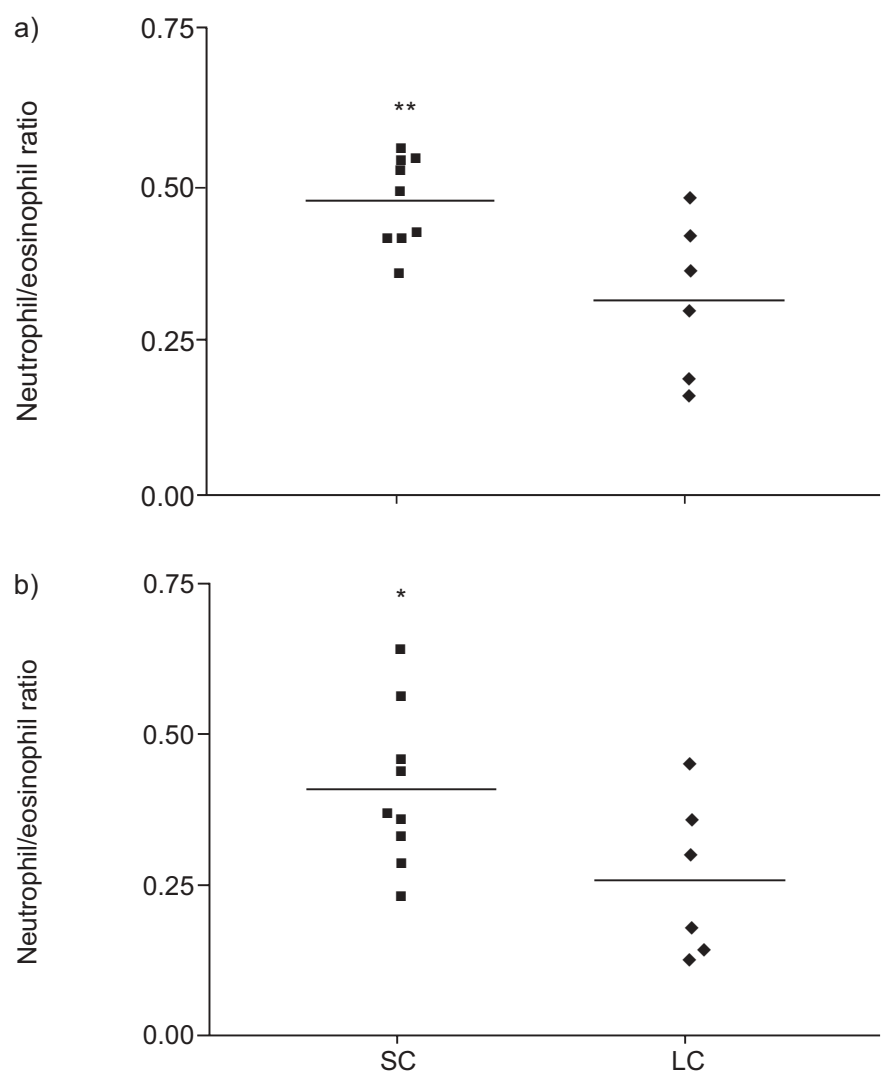

FIGURE 2. Dot plots showing neutrophil/eosinophil ratio in the a) inner and

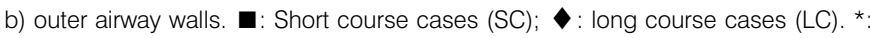
$p<0.05 * *: p<0.01$ 


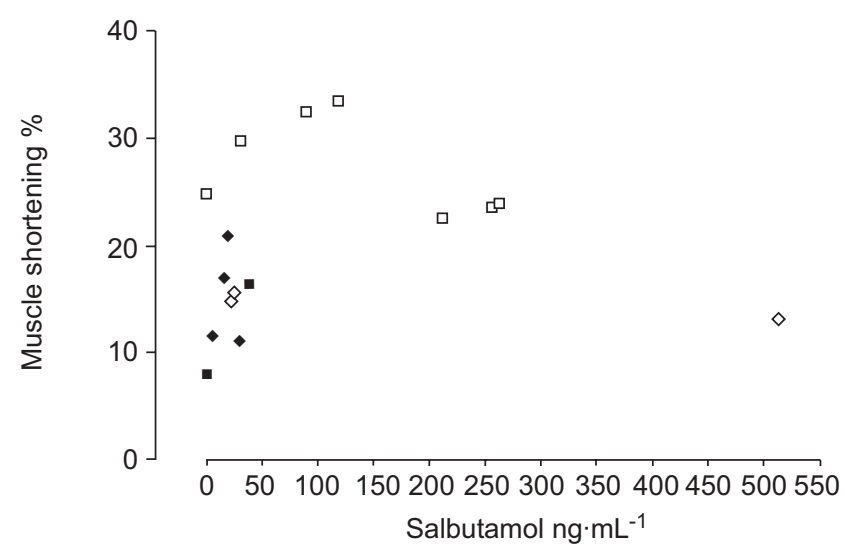

FIGURE 3. Dot plot showing salbutamol levels and muscle shortening percentage. $\square$ : short course (SC) cases with a mucus occupying ratio $<20 \%$ of the airway lumen; $\diamond$ : SC cases with a mucus occupying ratio $>20 \%$ of the airway lumen; $\mathbf{\square}$ : long course (LC) cases with a mucus occupying ratio $<20 \%$ of the airway lumen; : LC cases with a mucus occupying ratio $>20 \%$ of the airway lumen.

with a long course. These observations of a dichotomous timing in the duration of fatal attacks of asthma have been observed in three previous studies of fatal asthma [8-10]. The present study is novel in using tissue from uninflated lungs and it was found that compared with long course cases, those with a short course had more ASM shortening, despite higher levels of serum salbutamol.

The use of these tissues enabled PMS and mucus occupying ratio to be examined with a greater degree of reliability, as post mortem inflation of the lungs is likely to influence these outcomes. However, small airways were predominantly examined and the effects of pathology (such as mucous gland hypertrophy) in large airways where mucus accumulation is also observed in fatal asthma could not be assessed [6, 7]. The current authors did not observe a significant difference in airway wall dimensions between the two groups in the present study. Although the numbers of cases in each of the two groups limited the ability to detect differences between them, no trends in markers of severity between the short course and long course cases were observed. A loss of elastic tissue and disruption of alveolar attachments around small airways in cases of fatal asthma [21] might contribute to increased shortening of stimulated smooth muscle, however, no significant difference in the number of alveolar attachments between the case groups was observed. This suggests that airway/parenchymal uncoupling is not a cause of the difference between the groups.

Increased shortening of airway smooth muscle in the short course cases were found despite increased serum levels of salbutamol, compared with the long course cases. There is no evidence of significant post mortem redistribution of salbutamol [22]. Salbutamol is not a lipophilic drug and metabolism of salbutamol ceases with hepatic blood flow. Although inhaled $\beta$-agonists have a good safety record, there are some data to suggest the possibility of increased cardiotoxicity of these agents in the presence of hypoxia [23]. The findings of different salbutamol levels related to time to death does not necessarily support a cardiotoxic effect, although if these medications exerted toxic effects the findings show these would be confined to the short course cases where serum levels were highest. The high levels $\left(>80 \mathrm{ng} \cdot \mathrm{mL}^{-1}\right)$ observed in the majority of short course cases were much greater than the serum value observed in children with acute asthma $\left(22.7 \mathrm{ng} \cdot \mathrm{mL}^{-1}\right)$ after administration of nebulised salbutamol [24]. The apparent lack of effect of salbutamol in these early cases may suggest tachyphylaxis in this group, which recently has been suggested to have a genetic basis [25]. Tachyphylaxis to excessive use of short acting $\beta$-agonists in a susceptible group could make them particularly vulnerable to acute severe bronchoconstriction.

The present study confirms the dichotomy of both time to death and the neutrophil/eosinophil ratio in cases of fatal asthma. METZGER et al. [11] observed in bronchoalveolar lavage (BAL) an influx of neutrophils and eosinophils into the airways following local allergen challenge. However, neutrophil levels had returned to normal by $96 \mathrm{~h}$, while eosinophil and helper Tcell numbers remained elevated. NOCKER et al. [26] also reported elevated numbers of neutrophils in BAL at $4 \mathrm{~h}$ following local allergen challenge. These observations suggest that if death occurs due to excessive airway narrowing following an acute inflammatory stimulus, the time to death will determine the observed neutrophil/eosinophil ratio. The current authors proposed that factors such as the severity of airway remodelling may determine the time to death [10]. However, this does not readily explain the clear distinction in time between the short and long course groups.

Increased thickness of the WAi is related (albeit nonlinearly) to excessive airway narrowing as the smooth muscle contracts [27]. One might expect a continuum of times to death as a result. This relationship might be affected by a number of confounders. These include the strength of the stimulus which may alter the tension developed in the smooth muscle, the expulsion of mucus into the airway lumen, the effects of treatment and the effects of patient variables such as obesity and other illness, especially in older subjects. These confounders might be expected to be distributed continuously. The dichotomy of times which is clearly evident in four different series of fatal asthma cases is, therefore, interesting. This might arise for a number of reasons. First, two different mechanisms of excessive airway narrowing (mucus accumulation versus PMS) with different time courses of action may cause excessive airway narrowing. Secondly, different stimuli such as viral infection or allergen may precipitate the fatal attack, but with different time courses. Thirdly, exposure to an inflammatory stimulus, especially allergen, may have a biphasic reaction with early and late phases [28]. This may correspond to the dichotomy of times to death in these studies of fatal asthma. In the present study, the findings of greater shortening of ASM in the short course cases and more mucus in the lumen in the long course cases support the first hypothesis. The consistent findings of increased eosinophils in the long course cases is compatible with the late phase reaction, however, the present authors were unable to assess this further in the case series.

It is possible that the strength of the stimulus associated with the fatal attack might determine the degree of airway PMS. It has been shown that increasing the stimulus strength can achieve excessive airway narrowing in subjects 
using long-acting $\beta$-agonists [29], and it is possible that exposure to a powerful bronchoconstricting stimulus (on a background of severe airway pathology) may have been the cause of death in the short course cases. Studies of rapid-onset and slow-onset attacks of near-fatal asthma have suggested that rapid-onset attacks may have different trigger factors and lower rates of infection than slow-onset attacks cases [30, 31]. Other studies have also alluded to allergen as a trigger factor for sudden attacks of asthma [32]. Due to the out-of-hospital deaths in the present cases and the reliance on second-hand reporting, it was difficult to assess the role of trigger factors in the present study. A possible trigger factor was identified in two $(33 \%)$ of the long course cases (respiratory tract infection in both) and in four (40\%) of the short course cases (exercise in two, exposure to birds in an aviary in one, and ingestion of prepackaged fried potato chips in one).

It is not clear why some patients die of asthma, although fatal attacks occur on a background of clinically severe disease and extensive airway remodelling. It seems likely that a combination of a strong bronchoconstricting and/or mucus-producing stimulus and severe airway remodelling are responsible. The reproducible observations, in four separate studies of post mortem tissues, of a dichotomy in times to death and in neutrophil/eosinophil ratios are intriguing.

In conclusion, the present study suggests a strong stimulus, which causes death through muscle shortening in short course cases (perhaps with $\beta$-agonist tachyphylaxis as an added risk factor) and possibly through accumulation of mucus in long course cases. Smooth muscle shortening and mucus secretion are related to mast cell degranulation. It is possible that a strong stimulus may lead to mast cell degranulation and that the time course of the fatal attack is then determined by two distinct and time-related processes, muscle shortening and mucus accumulation.

\section{ACKNOWLEDGMENTS}

The police statements and autopsy reports for cases were made available by the office of the Victorian State Coroner G. Johnstone. Salbutamol assays were performed by F. Couper under the supervision of O. Drummer at the Victorian Institute of Forensic Medicine (Australia). P. Jessup provided helpful pathological advice.

\section{REFERENCES}

1 Laitinen A, Laitinen LA. Cellular infiltrates in asthma and in chronic obstructive pulmonary disease. Am Rev Respir Dis 1991; 143: 1159-1161.

2 Carroll N, Elliot J, Morton A, James A. The structure of large and small airways in nonfatal and fatal asthma. Am Rev Respir Dis 1993; 147: 405-410.

3 Carroll N, Cooke C, James A. The distribution of eosinophils and lymphocytes in the large and small airways of asthmatics. Eur Respir J 1997; 10: 292-300.

4 Stewart AG. Airway wall remodelling and hyperresponsiveness: modelling remodelling in vitro and in vivo. Pulm Pharmacol Ther 2001; 14: 255-265.

5 Moreno RH, Hogg JC, Pare PD. Mechanics of airway narrowing. Am Rev Respir Dis 1986; 133: 1171-1180.
6 Huber HL, Koessler KK. The pathology of fatal asthma. Arch Intern Med 1922; 30: 689-760.

7 Dunnill MS. The pathology of asthma, with special reference to changes in the bronchial mucosa. J Clin Path 1960; 13: 27-33.

8 Azzawi M, Johnston PW, Majumdar S, Kay AB, Jeffery PK. $\mathrm{T}$ lymphocytes and activated eosinophils in airway mucosa in fatal asthma and cystic fibrosis. Am Rev Respir Dis 1992; 145: 1477-1482.

9 Sur S, Crotty TB, Kephart GM, et al. Sudden-onset fatal asthma. A distinct entity with few eosinophils and relatively more neutrophils in the airway submucosa?. Am Rev Respir Dis 1993; 148: 713-719.

10 Carroll N, Carello S, Cooke C, James A. Airway structure and inflammatory cells in fatal attacks of asthma. Eur Respir J 1996; 9: 709-715.

11 Metzger WJ, Zavala D, Richerson HB, et al. Local allergen challenge and bronchoalveolar lavage of allergic asthmatic lungs. Description of the model and local airway inflammation. Am Rev Respir Dis 1987; 135: 433-440.

12 Crane J, Pearce N, Flatt A, et al. Prescribed fenoterol and death from asthma in New Zealand, 1981-83: case-control study. Lancet 1989; 1: 917-922.

13 Spitzer WO, Suissa S, Ernst P, et al. The use of beta-agonists and the risk of death and near death from asthma. $N$ Engl J Med 1992; 326: 501-506.

14 Garrett JE, Lanes SF, Kolbe J, Rea HH. Risk of severe life threatening asthma and beta agonist type: an example of confounding by severity. Thorax 1996; 51: 1093-1099.

15 Abramson MJ, Bailey MJ, Couper FJ, et al. Are asthma medications and management related to deaths from asthma? Am J Respir Crit Care Med 2001; 163: 12-18.

16 Campbell DA, McLennan G, Coates JR, et al. Near fatal asthma attacks: the reliability of descriptive information collected from close acquaintances. Thorax 1993; 48: 1099-1104.

17 Couper FJ, Drummer OH. Gas chromatographic-mass spectrometric determination of beta 2-agonists in post mortem blood: application in forensic medicine. J Chromatogr B Biomed Appl 1996; 685: 265-272.

18 James AL, Hogg JC, Dunn LA, Pare PD. The use of the internal perimeter to compare airway size and to calculate smooth muscle shortening. Am Rev Respir Dis 1988; 138: 136-139.

19 Aikawa T, Shimura S, Sasaki H, Ebina M, Takishima T. Marked goblet cell hyperplasia with mucus accumulation in the airways of patients who died of severe acute asthma attack. Chest 1992; 101: 916-921.

20 Carroll N, Lehmann E, Barret J, Morton A, Cooke C, James A. Variability of airway structure and inflammation in normal subjects and in cases of nonfatal and fatal asthma. Pathol Res Pract 1996; 192: 238-248.

21 Mauad T, Silva LF, Santos MA, et al. Abnormal alveolar attachments with decreased elastic fiber content in distal lung in fatal asthma. Am J Respir Crit Care Med 2004; 170: 857-862.

22 Couper FJ, Drummer OH. Post mortem stability and interpretation of beta 2-agonist concentrations. J Forensic Sci 1999; 44: 523-526. 
23 Bremner P, Burgess CD, Crane J, et al. Cardiovascular effects of fenoterol under conditions of hypoxaemia. Thorax 1992; 47: 814-817.

24 Penna AC, Dawson KP, Manglick P, Tam J. Systemic absorption of salbutamol following nebulizer delivery in acute asthma. Acta Paediatr 1993; 82: 963-966.

25 Israel E, Chinchilli VM, Ford JG, et al. Use of regularly scheduled albuterol treatment in asthma: genotype-stratified, randomised, placebo-controlled cross-over trial. Lancet 2004; 364: 1505-1512.

26 Nocker RE, Out TA, Weller FR, Mul EP, Jansen HM, van der Zee JS. Influx of neutrophils into the airway lumen at $4 \mathrm{~h}$ after segmental allergen challenge in asthma. Int Arch Allergy Immunol 1999; 119: 45-53.

27 James AL, Pare PD, Hogg JC. The mechanics of airway narrowing in asthma. Am Rev Respir Dis 1989; 139: 242-246.

28 Cockcroft DW, Murdock KY. Comparative effects of inhaled salbutamol, sodium cromoglycate, and beclomethasone dipropionate on allergen-induced early asthmatic responses, late asthmatic responses, and increased bronchial responsiveness to histamine. J Allergy Clin Immunol 1987; 79: 734-740.

29 Bel EH, Zwinderman AH, Timmers MC, Dijkman JH, Sterk PJ. The protective effect of a beta 2 agonist against excessive airway narrowing in response to bronchoconstrictor stimuli in asthma and chronic obstructive lung disease. Thorax 1991; 46: 9-14.

30 Rodrigo GJ, Rodrigo C. Rapid-onset asthma attack: a prospective cohort study about characteristics and response to emergency department treatment. Chest 2000; 118: 1547-1552.

31 Plaza V, Serrano J, Picado C, Sanchis J. Frequency and clinical characteristics of rapid-onset fatal and near-fatal asthma. Eur Respir J 2002; 19: 846-852.

32 O'Hollaren MT, Yunginger JW, Offord KP, et al. Exposure to an aeroallergen as a possible precipitating factor in respiratory arrest in young patients with asthma. $N$ Engl J Med 1991; 324: 359-363. 